\title{
WHITE-SPACE DETECTION TECHNIQUES BASED ON NEIGHBORHOOD DiSTANCE MEASUREMENTS
}

\author{
BOIANGIU, C. A.; CANANAU, D. C.; \\ DVORNIC, A. I. \& SPATARU, A. C.
}

Abstract: The purpose of this paper is to present a model for white space separators detection by using simple mathematical algorithms based on distances or font measure. The algorithms present new features as they are independent of the position in page, but they start from common ideas and improve them in order to obtain a satisfactory output.

Key words: white-spaces, neighbourhood distance, crosshair, digitization
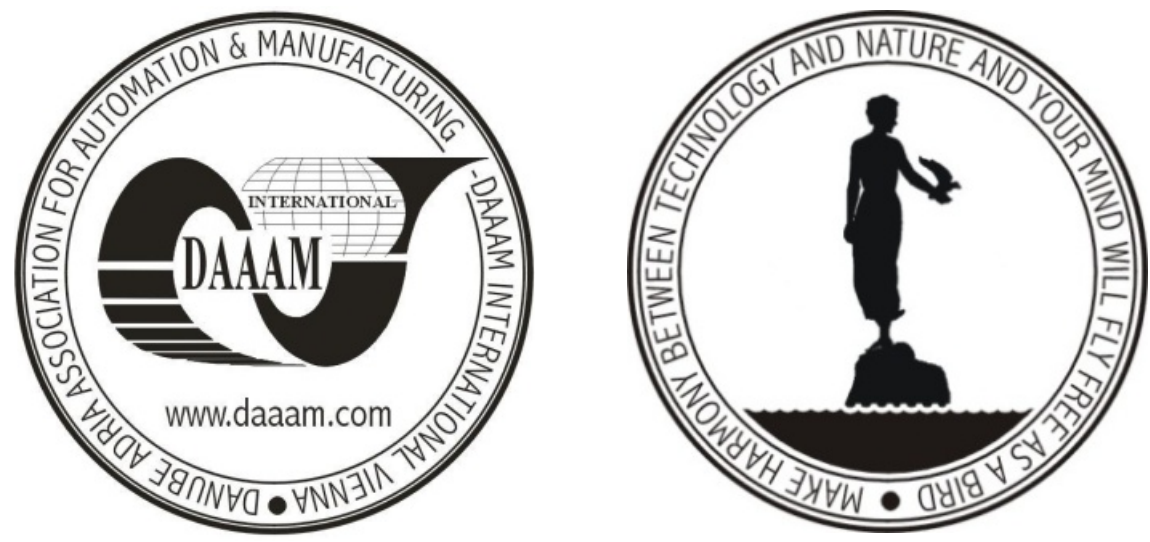

Authors' data: Lecturer $\mathrm{PhD}$. Eng. Boiangiu, C[ostin] A[nton]; Eng. Cananau, D[an] C[ristian]; Eng. Dvornic A[ndrei] I[ulian]; Eng. Spataru A[ndrei] C[ristian], University „Politehnica“ of Bucharest, Splaiul Independentei 313, Bucharest, Postal Code 060042, Romania, costin.boiangiu@cs.pub.ro, dan_cananau@yahoo.com, andrei.dvornic@yahoo.co.uk, andrei.spataru@yahoo.com

This Publication has to be referred as: Boiangiu, C[ostin] A[nton]; Cananau, D[an] C[ristian]; Dvornic A[ndrei] I[ulian] \& Spataru A[ndrei] C[ristian] (2009). WhiteSpace Detection Techniques Based on Neighborhood Distance Measurements, Chapter 32 in DAAAM International Scientific Book 2009, pp. 305-314, B. Katalinic (Ed.), Published by DAAAM International, ISBN 978-3-901509-69-8, ISSN 17269687, Vienna, Austria

DOI: 10.2507/daaam.scibook.2009.32 